\title{
Liver organoids reproduce alpha-1 antitrypsin deficiency-related liver disease
}

\author{
Gema Gómez-Mariano ${ }^{1}$ - Nerea Matamala ${ }^{1}$ - Selene Martínez ${ }^{1}$. lago Justo ${ }^{2}$ - Alberto Marcacuzco ${ }^{2}$ - Carlos Jimenez ${ }^{2}$. \\ Sara Monzón ${ }^{3} \cdot$ Isabel Cuesta ${ }^{3} \cdot$ Cristina Garfia ${ }^{4} \cdot$ María Teresa Martínez $z^{5} \cdot$ Meritxell Huch $^{6} \cdot$ Ignacio Pérez de Castro $^{7}$. \\ Manuel Posada ${ }^{8} \cdot$ Sabina Janciauskiene $^{9} \cdot$ Beatriz Martínez-Delgado ${ }^{1,8} \mathbb{C}$
}

Received: 30 August 2019 / Accepted: 26 November 2019 / Published online: 13 December 2019

(c) The Author(s) 2019

\begin{abstract}
Background and aims Alpha-1 antitrypsin (AAT) is a product of SERPINA1 gene mainly expressed by hepatocytes. Clinically relevant mutations in the SERPINAl gene, such as Z (Glu342Lys), results in an expression of misfolded AAT protein having high propensity to polymerize, accumulate in hepatocytes and thus to enhance a risk for hepatocyte damage and subsequent liver disease. So far, the relationship between the Z-AAT accumulation and liver cell damage remains not completely understood. We present three-dimensional organoid culture systems, as a novel tool for modeling Z-AAT-related liver diseases. Methods We have established liver organoids from liver biopsies of patients with homozygous (ZZ) and heterozygous (MZ) deficiency and normal (MM) genotypes of AAT. The features of these organoid models were characterized by analyzing AAT protein secretion and intracellular aggregation in $\mathrm{MZ}$ and ZZ genotypes as well as SERPINAI expression in differentiated cultures.

Results Transcriptional analysis of differentiated organoid cultures by RNA-Seq showed hepatocyte-specific gene expression profile. Genes, such as $A L B, A P O B, C Y P 3 A 4$ and SERPINA1, were validated and confirmed through quantitative-PCR analysis. The organoids from MZ and ZZ cases showed intracellular aggregation and lower secretion of AAT protein, and lower expression of $A L B$ and $A P O B$, as typically seen in hepatocytes from Z-AAT deficiency patients. Furthermore, organoids responded to external stimulus. Treatment with oncostatin M, a well-known inducer of SERPINA1, increased expression of the full-length transcripts (AAT-1C) as well as the short transcript of AAT (AAT-ST1C4).

Conclusions Liver organoid model recapitulates the key features of Z-AAT deficiency and provides a useful tool for disease modeling.
\end{abstract}

Keywords Organoids $\cdot$ Liver $\cdot$ Alpha-1 antitrypsin deficiency $\cdot$ Oncostatin M $\cdot$ RNA-seq $\cdot$ SERPINA1

\section{Introduction}

Alpha1-antitrypsin (AAT), a prototypic member of the serine protease inhibitor (serpin) superfamily, is mainly produced by hepatocytes. A normal concentration of the AAT in

Gema Gómez-Mariano and Nerea Matamala contributed equally to this work

Electronic supplementary material The online version of this article (https://doi.org/10.1007/s12072-019-10007-y) contains supplementary material, which is available to authorized users.

Beatriz Martínez-Delgado

bmartinezd@isciii.es

Extended author information available on the last page of the article human blood ranges between 1 and $2 \mathrm{~g} / \mathrm{l}$ and it is estimated that liver accounts for about $80 \%$ of the total circulating AAT [1]. Human AAT is also an acute phase glycoprotein with broad anti-protease and anti-inflammatory functions, and therefore AAT deficiency leads to various health problems, including liver disorders [2].

AAT is encoded by the SERPINAI gene located at the distal end of the long arm of chromosome 14. The typical SERPINA1 gene consists of two alleles, named $\mathrm{M}$, which are responsible for the synthesis of quantitatively and qualitatively normal AAT. The most frequent deficient alleles are so called S (Glu264Val) and Z (Glu342Lys). The combinations of the $\mathrm{M}, \mathrm{S}$ and $\mathrm{Z}$ alleles give rise to the different genotypes MM, SS, MZ, SZ and ZZ. The homozygous ZZ genotype is the most relevant genotype in the clinical and 
genetic knowledge of SERPINA1, which results in about 90\% reduced levels of circulating AAT protein. The deficiency in $\mathrm{ZZ}$ cases occurs due to the aberrant folding of the Z-AAT causing its polymerization and intracellular accumulation. The clinical manifestations of severe AAT deficiency include liver (intracellular retention of aggregated AAT that resists degradation) and lung (missing protective levels of functional AAT) diseases, and less frequently skin diseases such as panniculitis or ANCA + vasculitis [3].

The AAT deficiency-related liver damage can occur at any age. Clinical studies have shown that children who progressed to the end-stage liver disease had more severe abnormalities in infancy such as persistent jaundice for more than 6 weeks, hepatomegaly, higher transaminases and severe morphological changes including bile duct reduplication, fibrosis and cirrhosis. Currently, however, there are no distinguishable features/markers allowing to predict which child will develop a fast decline in liver function requiring liver transplantation or who will recover without sequelae of chronic liver disease [4]. In adults, liver damage can be manifested by liver fibrosis and cirrhosis, and hepatocellular carcinoma [3, 5]. On the other hand, Z-AAT deficiency carriers may remain clinically healthy until later adulthood. This variability in clinical presentation suggests that in addition to inherited abnormality in AAT protein, other environmental, genetic and epigenetic factors are necessary to promote the development of the AAT deficiency-related liver disease. Therefore, better understanding of the molecular mechanisms underlying liver disease related to Z-AAT deficiency is of critical importance for the diagnosis and the development of specific and personalized therapies.

Currently, experimental studies investigating liver disease in AAT deficiency are limited by the difficulty to obtain human liver tissue and to maintain primary cultures of human hepatocytes. Alternatively, human embryonic stem cells and induced pluripotent stem cells are used [6]. However, full differentiation of stem cells into mature hepatocytes has yet not been reported.

Organoids are new three-dimensional (3D) model systems referred to a group of cells growing in a 3D structure that are generated from primary tissues or cells, with selfrenewal and self-organization capacity, maintaining similar appearance and functionality as the original tissue. Adult tissue-derived organoids can be maintained through indefinite passage and preserve genetic stability [7]. Recently, human liver organoids started to be used for the studies of various liver diseases $[8,9]$. The first described human liver organoids allowed the expansion of adult liver stem cells and subsequent differentiation to hepatocytes that recapitulate some function of ex vivo liver tissue. Moreover, differentiated liver organoids from AAT-deficient patients mimicked the characteristics of the disease [7]. In this study, we have established and compared adult human liver organoids from liver biopsies of individuals with normal, $\mathrm{MM}$ and deficient ZZ and MZ AAT genotypes. The aim was to show if liver organoid cultures can recapitulate the typical features of liver cells expressing normal and deficient AAT and can be useful for AAT deficiency-related liver disease modeling. Typical features of AAT deficiency-associated liver disease were analyzed in terms of AAT polymerization and secretion, and transcriptional induction of SERPINA1 gene transcripts in organoids subjected to external stimuli. The results show that liver organoids is a useful tool allowing modeling liver disease in individuals with different AAT mutations.

\section{Materials and methods}

\section{Patients and genotyping}

Organoids were established from liver biopsies collected from patients and controls at the Hospital 12 de Octubre in Madrid (Spain) and also provided by Dr. Huch at Cambridge University (UK). The ZZ organoids were derived from ZZ AATD patients with hepatic failure who had liver transplant, whereas $\mathrm{MZ}$ organoids were obtained from an adult MZ AATD patient who underwent colicestomy. The control MM AAT organoids were derived from an individual with hepatocellular carcinoma undergoing surgical resection. Tissue sample was obtained from macroscopically defined nonneoplastic adjacent area. All biopsies were genotyped for SERPINA1. Sequencing of SERPINA1 gene coding exons was performed by using previously described primers [10, 11] in an automatic sequencer (ABI PRISM 377 Applied BioSystems). Signed informed consent for the study was obtained from all the subjects and the research was approved by the ethics committee of Instituto de Salud Carlos III, Madrid, Spain.

\section{Establishment and culture of human liver organoids}

We aimed to generate liver organoids expressing $\mathrm{ZZ}$ and MZ genotypes of AAT, and normal MM variant of AAT. Following the protocol described by Huch and collaborators $[7,12]$, we established liver organoids from ductal cells of human liver. Briefly, after surgical excision, the tissue was kept cold in basal medium (Advanced DMEM/F12, 1\% penicillin/streptomycin, $1 \%$ Glutamax, $10 \mathrm{mM}$ Hepes) until processing. The tissue was minced, washed with cold medium (DMEM, Glutamax, DGlu, Pyruvato, $1 \%$ FBS, $1 \%$ penicillin/streptomycin) and digested (Earle's Balance Salt Solution (EBSS), collagenase D $2.5 \mathrm{mg} / \mathrm{ml}$, DNAse I $0.1 \mathrm{mg} / \mathrm{ml}$ ). After centrifugation, cells were resuspended in a basement matrix and seeded in a 24-well plate with isolation medium (basal medium supplemented with $25 \mathrm{ng} / \mathrm{ml}$ recombinant human Nogging, 30\% conditioned medium with Wnt3a 
and $10 \mu \mathrm{M}$ Rho kinase (Rock inhibitor)). In this specific culture condition, only progenitor adult stem cells, mainly located in ducts, are able to grow forming self-renewing organoids in the form of spheres. In each passage, organoids were removed from the basement matrix and transferred to fresh matrix. For expansion, the undifferentiated organoids were cultured in an expansion medium (EM) (basal medium, $1 \mathrm{mM} \mathrm{N}$-acetylcysteine, 5\% conditioned medium Rspo1, $10 \mathrm{mM}$ nicotinamide, $10 \mathrm{nM}$ recombinant human gastrin I, $50 \mathrm{ng} / \mathrm{ml}$ recombinant EGF, $100 \mathrm{ng} / \mathrm{ml}$ recombinant human fibroblast growth factor (FGF) $10,50 \mathrm{ng} / \mathrm{ml}$ recombinant human hepatocyte growth factor (HGF), supplemented with $10 \mu \mathrm{M}$ Rho kinase (Rock inhibitor)). The differentiation into hepatocytes was achieved with a differentiation medium (DM) (basal medium, 1:50 B27 supplement with or without vitamin A, 1:100 N2 supplement, $1 \mathrm{mM} N$-acetylcysteine, $10 \mathrm{nM}$ recombinant human-gastrin I, $50 \mathrm{ng} / \mathrm{ml}$ recombinant mouse EGF, $100 \mathrm{ng} / \mathrm{ml}$ recombinant human FGF10, $50 \mathrm{nM}$ A83-01, 10uM $\gamma$-secretase inhibitor (DAPT). Figure 1 illustrates different stages of the isolation, expansion and differentiation of the liver organoid.

\section{Identification of Z-AAT aggregates by using PAS-D staining}

To detect intracellular aggregates of the Z-AAT protein, cytospins of organoid liver cells were stained with the periodic acid-Schiff-diastase (PAS-D). Organoids were washed with phosphate-buffered saline (PBS), spread on slides (1 min at $600 \mathrm{rpm}$ ) and fixed with 95\% ethanol for $5 \mathrm{~min}$. Afterward, cells were washed twice with PBS and stained with hematoxylin-eosin and PAS-D.

\section{Detection of intracellular AAT by immunofluorescence}

Organoids growing in EM (undifferentiated) for 1-2 months or differentiated organoids growing in DM for 2 weeks were collected for immunofluorescence detection of total AAT or polymers of AAT. Cytospins of organoid cells were washed with PBS, fixed with $95 \%$ ethanol and permeabilized with $0.1 \%$ Triton X-100/PBS and blocked with $1 \%$ bovine serum albumin/phosphate-buffered saline (BSA/PBS). The expression of AAT protein was analyzed by incubating with antiAAT B9 antibodies (sc-59438 Santa Cruz Biotechnology, Santa Cruz, CA, USA) for total AAT and with D11 antibody specific for AAT polymers (provided by Sabina Janciauskiene, Hannover). A secondary anti-mouse FICT IGG (Sigma F0257) antibody was used. 4', 6-diamidino-2-phenylindole (DAPI Sigma) staining of cell nuclei was performed. The preparations were examined under a fluorescence microscope Zeiss Ax10. The proportion of positive cells showing AAT polymers (D11) was estimated by counting at least 300 nuclei from three different fields from MM, MZ and $\mathrm{ZZ}$ organoids (counting was performed by two independent researchers).

\section{Western blot analysis of AAT}

Native and polymeric forms of AAT protein were detected in cell pellets and culture medium of expanding and differentiated organoids. Cell pellets were lysed with RIPA buffer containing a cocktail of complete protease inhibitors (Roche, Mannhein, Germany). The lysates and culture media were electrophoresed on $10 \%$ polyacrylamide sodium dodecyl sulfate PAGE (SDS-PAGE). The insoluble fraction was sonicated and separated on non-denaturing 8\% PAGE. Proteins were detected with the following primary antibodies: antiAAT B9 (1:100 dilution) and anti-beta-actin (AC-74 Sigma Aldrich, Taufkirchen, Germany) (1:5000 dilution) followed by chicken anti-mouse IgG-HRP (sc-2954 Santa Cruz Biotechnology) (1:5000 dilution), and visualized after labeling with Immobilon HRP substrate (Millipore) using Chemidoc Touch imaging system (Biorad, Hercules, CA, USA). Quantification of the western blot bands of AAT protein of MM, $\mathrm{MZ}$ and $\mathrm{ZZ}$ organoids was performed with Image $\mathbf{J}$ software.

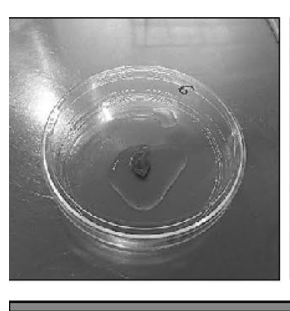

a Liver biopsy

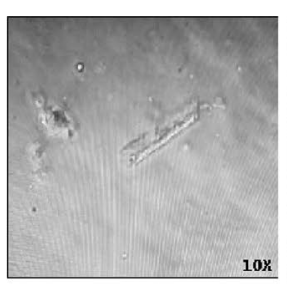

b Duct isolation

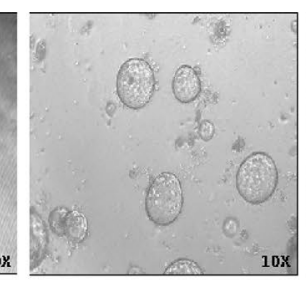

c Organoid in EM [10 days]
Fig. 1 Representative images of the expansion and differentiation of liver organoids. a Human liver organoids were obtained from a liver biopsy of patients. b Ductal cells are isolated, c, d Liver organoids are developed by growing in an expansion medium (EM) and a 3D

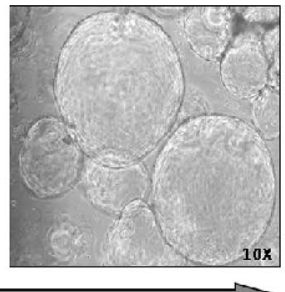

d Organoid in EM [20 days]

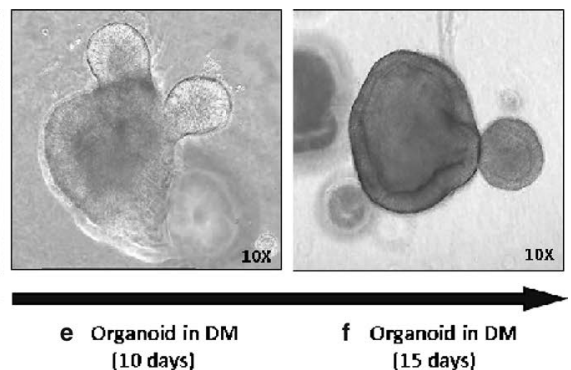

Matrigel, e, f Cultured in differentiation medium (DM) giving rise to differentiated organoids (magnification $\times 10$ ). Microscope Leica DMIL LED, camera Leica MC170HD 


\section{Transcriptomic analysis of liver organoids (RNA-Seq)}

Total RNA was isolated from organoid cells using TriReagent (Sigma) followed by DNase1 digestion step to ensure that the samples were not contaminated with genomic DNA. The purity of RNA was assessed using Agilent RNA 6000 Nano Kit and the Agilent 2100 Bioanalyzer. TruSeq Stranded mRNA Kit (Illumina) was used for library preparation based on the recommendations from the manufacturer. The sequencing of the library was performed at the Genomics service (ISCIII) on a NextSeq 500 sequencer using 75 base read lengths in paired-end mode. The obtained RNA-Seq data were analyzed by the Bioinformatics Facility (ISCIII). First, quality control analysis involving fastQC v0.11.3 (http://www.bioinformatics.babraham.ac.uk/proje cts/fastqc/) was carried out, and any adapter sequences as well as low quality $3^{\prime}$ ends were removed using Trimmomatic v0.36. The high-quality reads were then mapped against $\mathrm{Hg} 38$ human genome using Tophat v2.0.14 and mapping quality control was performed using RseQC v2.6.4. Transcriptome prediction and gene/isoform quantification was calculated using Cufflinks v2.2.1 based on Hg38 RefSeq reference genes. Finally, differential expression analysis was carried out using cuffdiff. Differentially expressed genes (DEGs) were considered when FDR values were $<0.05$. The CummeR bund R package (v2.14.0) was used for quality control and results visualization. The functional analysis was carried out with Functional Annotation Tool DAVID Bioinformatics Resources 6.8, NIAID/NIH.

\section{Validation of differentiation markers by RT-qPCR}

Progenitor and ductal cell markers, leucine-rich repeat containing $\mathrm{G}$ protein-coupled receptor 5 (LGR5) and keratin 19 (KRT19), and differentiation markers (albumin, $A L B$; apolipoprotein B, APOB; and cytochrome P450 3A4, CYP3A4) were analyzed by RT-qPCR. Total RNA was extracted from organoids by using TriReagent (Sigma) and expression was also analyzed in HepG2 cells and control liver biopsy. cDNA was synthesized using Maxima First Strand cDNA Synthesis kit (Thermo Scientific, Fermentas Life Sciences, St. LeonRot, Germany). The RT-qPCR of selected hepatocyte markers was performed in triplicate using Taqman Fast Advance master mix (Applied BioSystems) and specific primers and probes (Supplementary Table 1). Taqman probes were from Universal probe library, UPL, (Roche): KRT19 (\#71), LGR5 (\#78), ALB (\#44), APOB (\#90), CYP3A4 (\#50). Amplification conditions were as follows: $95{ }^{\circ} \mathrm{C}$ for $20 \mathrm{~s}, 45$ denaturation cycles at $95^{\circ} \mathrm{C}$ for $3 \mathrm{~s}$, annealing at $60^{\circ} \mathrm{C}$ for $30 \mathrm{~s}$. RT-qPCR was performed on the QuantStudio 5 System (ThermoFisher Scientific) and the analysis using the software QuantStudio Design and Analysis Software v1.4.3. Glyceraldehyde-3-phosphate dehydrogenase (GADPH) was used as an endogenous control. The relative gene expression was calculated by comparative $\mathrm{Ct}$ method and obtaining the fold-change value (DDCt).

\section{Expression analysis of SERPINA1 transcripts}

The expression of alternative SERPINA1 gene transcripts was analyzed in RNA from MM, MZ and ZZ organoids under both the expansion (EM) and differentiation (DM) conditions. The RT-qPCR for SERPINA1 transcripts, generated by the use of different promoters and alternative splicing between non coding exons $1 \mathrm{~A}, 1 \mathrm{~B}$ and $1 \mathrm{C}$, was performed with primers and methods previously described [13]. In addition, expression of SERPINA 1 short transcripts was analyzed as described [14].

\section{Exogenous organoid stimulation with OSM}

To study the expression regulation of SERPINAl gene, organoids were exposed to oncostatin M (OSM: rhOncostatin $M$ recombinant human $E$. Coli from RD Systems 295 $\mathrm{OM})$. We added OSM $(50 \mathrm{ng} / \mathrm{ml})$ to organoids at day $14 \mathrm{of}$ the differentiation process. After $16 \mathrm{~h}$ of stimulation, cells were collected and the expression of SERPINAI transcripts was quantified. A Student's $t$ test analysis was performed to compare the gene expression between controls and stimulated cells. Statistically significant values were considered if $p<0.05$.

\section{Results}

\section{Hepatocyte-specific gene expression in differentiated organoids}

Transcriptome analysis by RNA-Seq was used to compare undifferentiated and differentiated liver organoids independently of the genotypes. As expected, a high number of differentially expressed genes DEGs (3097 genes) were found between the undifferentiated and differentiated organoids. The top hundred most differentially expressed genes are illustrated in Fig. 2a, which include 48 up-regulated and 53 down-regulated genes after differentiation. Highly induced genes included typical mature hepatocyte genes, as previously described by other authors [15, 16]. The results confirmed that differentiated organoids, regardless of the AAT genotype, show increased expression of hepatocyte-specific genes such as albumin $(A L B)$, apolipoprotein B (APOB), apolipoprotein $\mathrm{C} 3$ (APOC3), cytochromes CYP3A4, CYP2C8 (cytochrome P450 family 2 subfamily $C$ member 8 ), as well as metabolism enzymes phospholipase A2 group XIIB (PLA2G12B), acyl-CoA thioesterase 12 (ACOT12), UDP glucuronosyltransferase 

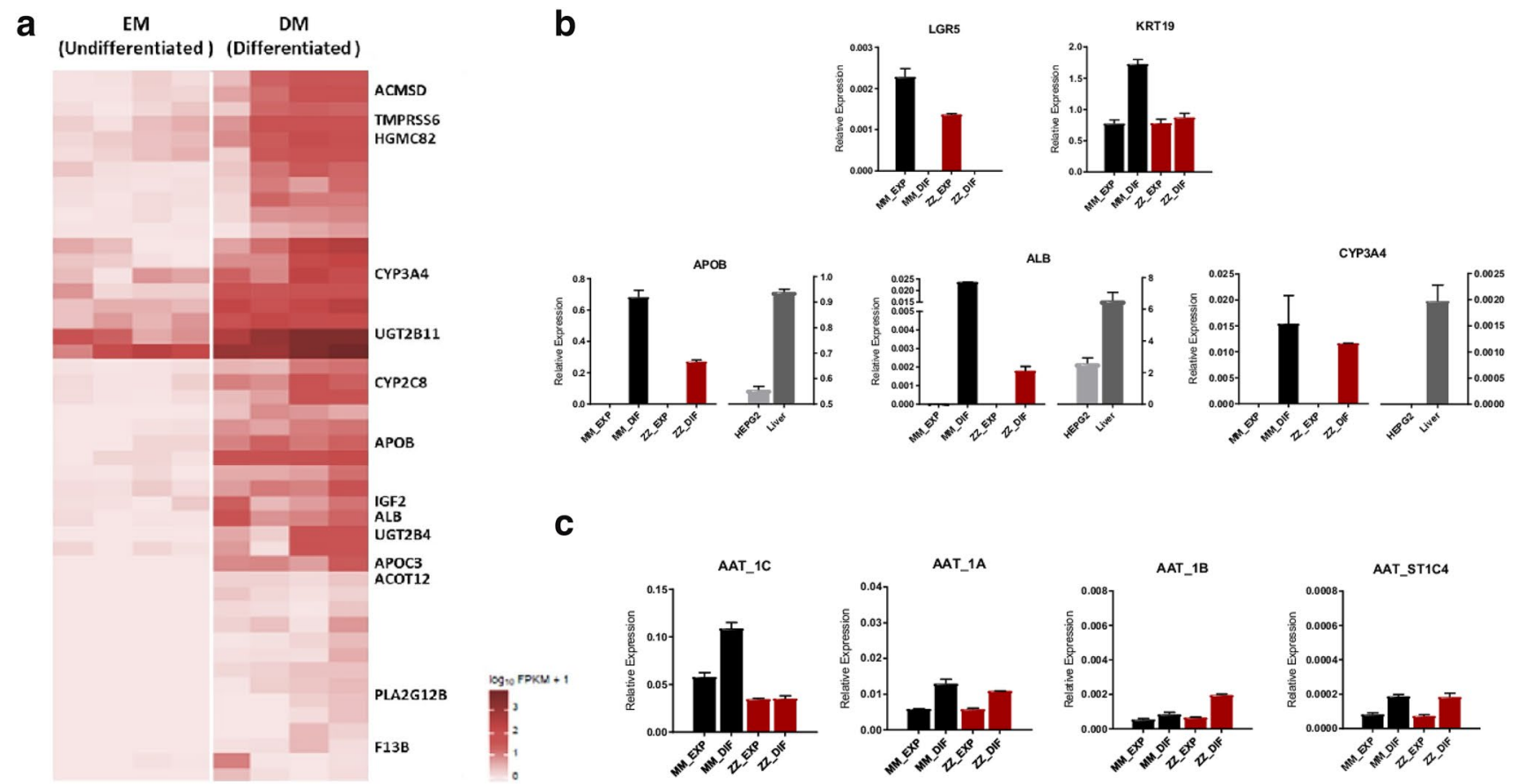

C
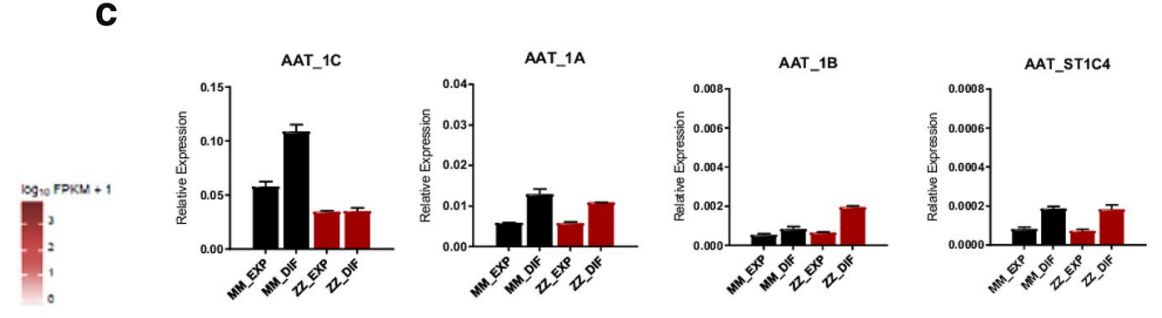

Fig. 2 Gene expression analysis in human liver organoids. a Heatmap of the top up-regulated differentially expressed genes between undifferentiated organoids (EM) and organoids differentiated into hepatocytes (DM). Some of the most relevant mature hepatocyte genes are represented at the right side. Darker color means higher expression levels. Gene expression analysis of differentiation markers in organoids MM (black) and $\mathrm{ZZ}$ (red) under expansion and differentiation conditions: b expression of markers of pluripotent (LGR5), ductal cells (KRT19) and differentiated hepatocytes $(A L B, A P O B$ and CYP3A4); $\mathbf{c}$ expression of alternative transcripts of SERPINA1 gene (1C, 1A and 1B) and short transcripts (ST1C4) in MM and ZZ organoids family 2 member B4 (UGT2B4), insulin-like growth factor 2 receptor $(I G F 2 R)$, UDP glucuronosyltransferase family 2 member B11(UGT2B11), 3-hydroxy-3-methylglutaryl-CoA synthase 2 (HMGCS2), transmembrane serine protease 6 (TMPRSS6), aminocarboxymuconate semialdehyde decarboxylase $(A C M S D)$ and coagulation factor XIII B chain $(F 13 B)$. A complete list of the top 100 most DEGs in differentiated organoids can be found in supplementary Tables 2 and 3.

The expression of certain progenitor, ductal and hepatocyte cell markers were validated in MM and ZZ by RTqPCR (Fig. 2b). As expected, both MM and ZZ organoids when growing in EM expressed markers of progenitor (LGR5) and ductal (KRT19) cell lineage, but these markers disappeared after differentiation. In contrast, organoids cultured in DM highly expressed hepatocyte genes, such as albumin $(A L B)$, apolipoprotein $\mathrm{B}(A P O B)$ and cytochrome P450 3A4 (CYP3A4), which were mostly not detected in undifferentiated liver organoid cultures. Remarkably, when compared to MM AAT organoids, lower expression of the $A P O B$ and $A L B$ hepatocyte markers, but increased expression of the CYP3A4, was observed in ZZ AAT organoids.

\section{SERPINA1 gene transcripts expression in liver organoids under expansion (EM) and differentiation (DM) conditions}

The expression of full-length and short (STIC4) transcripts of SERPINA1 gene [14] was analyzed in MM and ZZ AAT-derived liver organoids (Fig. 2c). Higher expression of all SERPINA1 transcripts was observed in differentiated than in undifferentiated $\mathrm{MM}$ and $\mathrm{ZZ}$ organoids, indicating that SERPINA1 gene is induced with the differentiation process in hepatocytes. Moreover, SERPINAI expression levels were higher in MM than in ZZ differentiated hepatocytes. Interestingly, in ZZ AAT organoids the expression of $1 \mathrm{C}$ transcript, the main liver transcript, did not increase during the differentiation process (Fig. 2c). Only a slight increase in the expression of the other minor AAT transcripts originated by the monocyte promoters (1A and 1B) was detected. A short STIC4 transcript of SERPINA 1 gene was induced in differentiated $\mathrm{M}$ and $\mathrm{Z}$ hepatocytes as compared to undifferentiated organoids. 


\section{Regulation of SERPINA1 gene transcripts by oncostatin M (OSM)}

The production of AAT in hepatocytes is controlled by a variety of cytokines released during inflammation, whereas leading regulators are IL-6-type cytokines. Therefore, we next analyzed transcriptional regulation of SERPINAl gene in liver organoids treated with oncostatin $\mathrm{M}$, a pleiotropic cytokine that belongs to the IL- 6 group of cytokines, a wellknown inducer of AAT production [17]. This analysis was performed on both undifferentiated (EM) and differentiated (DM) organoids from MM, MZ and ZZ AAT cases (Fig. 3). In MM AAT organoids, the $1 \mathrm{C}$ full-length transcript of AAT, which is typically expressed by hepatocytes, was induced by OSM under both EM and DM conditions. However, in ZZ organoids, the more evident induction of $1 \mathrm{C}$ transcript was observed in the differentiated stage. It is important to point out that in differentiated hepatocytes, OSM also induced the expression of transcripts derived from the monocyte promoter 1A and 1B (Supplementary Fig). Short transcripts ST1C4 were highly induced by OSM in differentiated MM and MZ AAT organoids, and to a lesser extent in ZZ AAT organoids (Fig. 3). No expression of the other described short transcript, ST1C5, was detected in the liver organoids.

\section{Detection of PAS-D-positive AAT polymers in liver organoids}

The presence of aberrant AAT polymers positive for PAS diastase-resistant (PAS-D) staining within hepatocytes is a characteristic feature of Z-AAT-deficient liver $[3,5,18]$. As expected, positive PAS-D staining was observed in organoids generated from the $\mathrm{ZZ}$ and from MZ AAT patients indicating the accumulation of the Z-AAT polymers within differentiated organoid cells. By contrast, PAS-D staining was negative in MM AAT organoids (Fig. 4).

\section{Detection of total and polymeric form of AAT protein in liver organoids}

To detect specifically AAT protein, we performed immunofluorescence assays in undifferentiated and differentiated organoids from MM, MZ and ZZ AAT cases (Fig. 5). As expected, Z-AAT polymers were evident in Z, but not in M organoids. In both $\mathrm{ZZ}$ and MZ cases, AAT polymers were limited to restricted groups of cells or isolated cells. The quantification of cells positive for AAT polymer staining (D11 antibody) revealed that MZ organoids had around 5\% of cells with polymer accumulation, approximately half of the positive cells found in ZZ organoids (10\%) (Fig. 5c).

The western blot analyses revealed the presence of AAT protein in the extracellular medium and cell extracts of MM and MZ differentiated organoids (Fig. 6). The monomeric
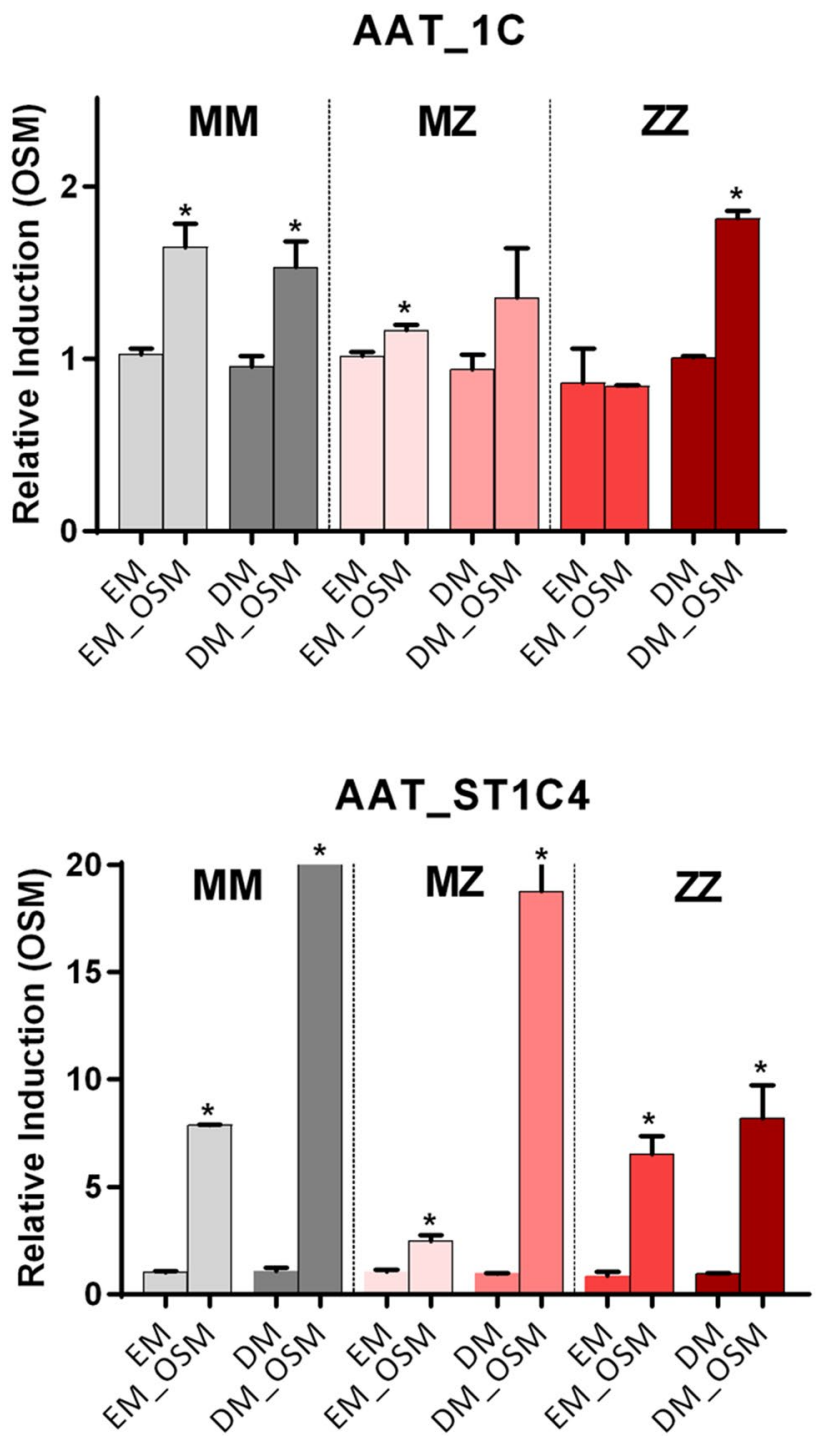

Fig. 3 Induction of SERPINA1 gene transcripts (1C) and short transcripts (ST1C4) by stimulations with OSM. Relative gene expression of the analyzed transcripts was measured in organoids grown in expansion medium (EM) and in differentiated organoids (DM). Statistically significant values are shown by $*(p<0.05)$

AAT protein was not detected in the cell extracts and extracellular medium of ZZ AAT organoids. By contrast, we found that the largest amount of insoluble AAT was present in $\mathrm{ZZ}$ compared to $\mathrm{MZ}$ or MM organoids (Fig. 6).

\section{Discussion}

Inherited AAT deficiency is a common cause of genetic liver disease having a wide spectrum of presentations ranging from neonatal liver disease to an incidental finding of adulthood end-stage cirrhosis requiring transplantation. Currently, there are no biomarkers/predictors to identify 

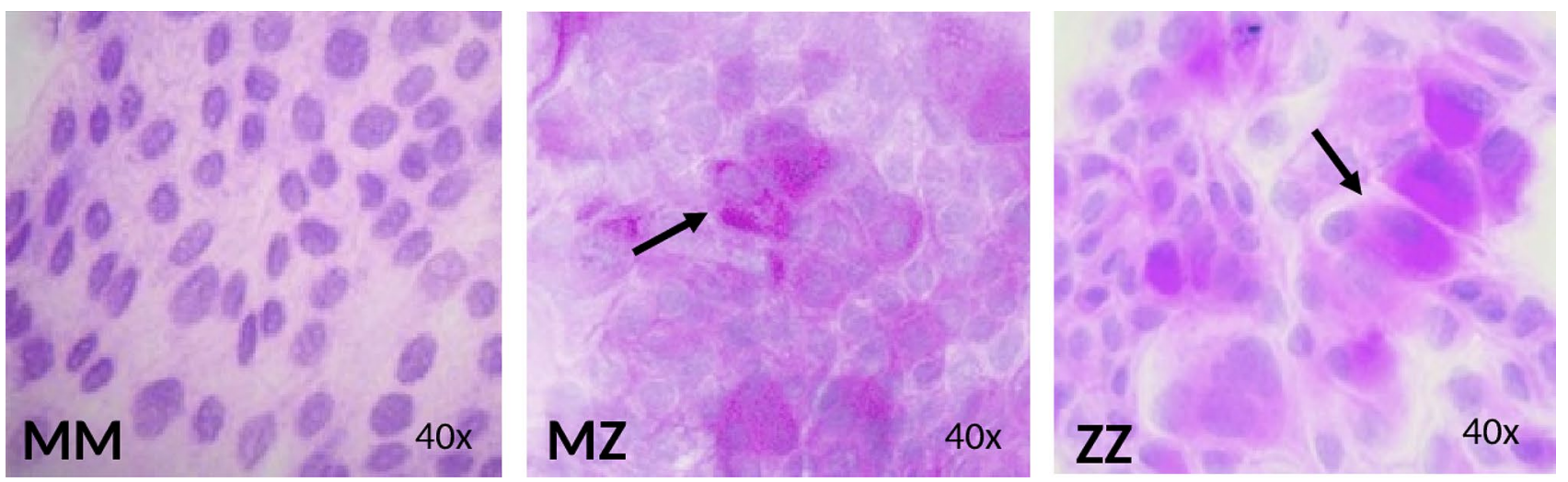

Fig. 4 PAS-D staining for the detection of Z-AAT protein aggregates. Differentiated hepatocytes from MM organoids did not show PAS+ staining, while the MZ and $\mathrm{ZZ}$ organoids show evident aggre-

AAT-deficient patients who will or not develop a severe course of liver disease. Moreover, the molecular mechanisms of AAT deficiency-related liver diseases remain largely unexplored.

Primary human hepatocytes reflect the metabolism and functionality of the human liver and are considered as the gold standard for hepatic ex vivo studies. However, the availability of primary human hepatocytes, especially from patients with rare inherited diseases such as AAT deficiency, is limited, and the function of isolated hepatocytes is hard to maintain ex vivo. When cultured in vitro, these cells undergo dedifferentiation, causing them to lose hepatocyte function. To solve the above issues, the development of new models allowing the culture and study of functional human hepatocytes is of great need [19-21].

To date, the 3D organoids are the best available representation of the liver cells with several advantages. First, organoids permit a long-term expansion with preserved genomic stability; second, organoid cultures recapitulate hepatocyte functions and allow to study molecular mechanisms of healthy and diseased liver, ex vivo; and third, organoids can be transplanted, as demonstrated in mouse models $[7,22]$, opening new avenues for regenerative medicine and gene therapy. These advantages of organoid systems together with emerging new technologies (such as 3D printing and imaging techniques) significantly contribute to the new strategies of how liver diseases are studied, and therapeutics developed.

Extensive analysis of cultured liver organoids revealed that the expanded cells preserve their genetic integrity over months in culture [7]. These results open up the avenue to start using human liver material expanded in vitro as an alternative hepatocyte source for studies of rare hereditary liver diseases. A variety of monogenic hereditary diseases affect the liver specifically, and these should be studied individually. gates of Z polymers. Microscope Leica DM 2000, camera Leica DFC450 and software LAS v4 (magnification $\times 40$ )

Therefore, in this study we established human liver organoid cultures reproducing the main characteristics of the wild-type $\mathrm{M}$ and pathogenic, deficient $\mathrm{ZZ}$ and $\mathrm{MZ}$ variants of AAT protein. When compared to 2D cell models [19], organoids are closer to liver tissue with respect to morphology, gene expression, and protein secretion. For example, the liver produces and secretes major proteins into the circulation, such as albumin, AAT, fibrinogens and apolipoproteins. Previous studies demonstrated that liver organoid cultures are able to express and secrete albumin and apolipoprotein B [7]. Patients with liver damage almost always have hypoalbuminemia caused by decreased albumin synthesis by the hepatocytes [23]. Data from a large cohort of patients revealed that ZZ AAT carriers have markedly reduced serum triglyceride and VLDL cholesterol concentrations compared to non-carriers [24]. In the model, we not only confirm that differentiated hepatocyte organoids express $A L B$, SERPINA1, CYP3A4 and APOB, but also demonstrate that organoids from ZZ AAT individuals have lower expression of $A L B$ and $A P O B$ in comparison to organoids from MM AAT. These findings further confirm that organoids from different genetic variants of AAT recapitulate specific features of the condition.

As an acute phase reactant protein, expression of AAT increases in response to various stimuli. Specifically, in the liver AAT is mainly regulated by IL-6-like cytokines including OSM. This process is mediated by the interaction between the hepatocyte promoter of SERPINAI and an OSM response element located at the $3^{\prime}$ UTR of the AAT gene via the interaction of transcription factors like STAT3 [25]. We found that OSM significantly up-regulates AAT expression in $\mathrm{MM}$ and $\mathrm{MZ}$ organoids under undifferentiated and differentiated stages, whereas in $\mathrm{ZZ}$ organoids AAT was up-regulated only after differentiation. Currently, we are not able to explain these latter differences; however, the results provide clear evidence that organoids represent a 


\section{a}
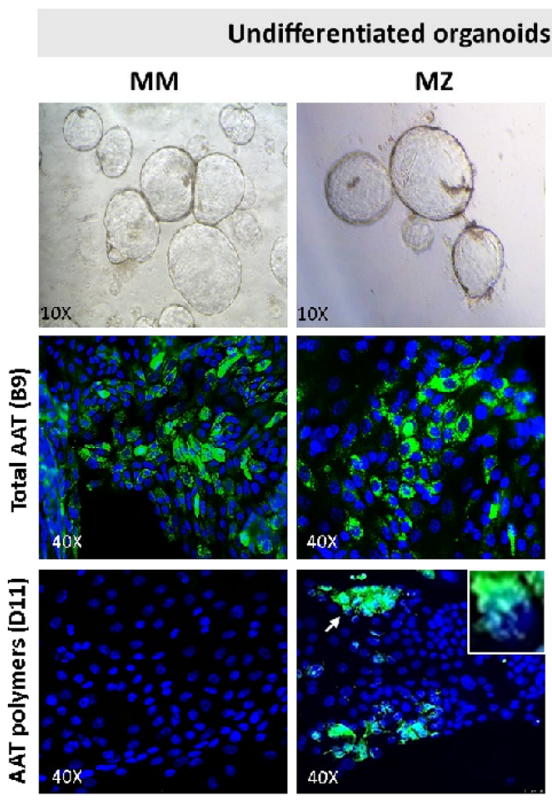

ZZ
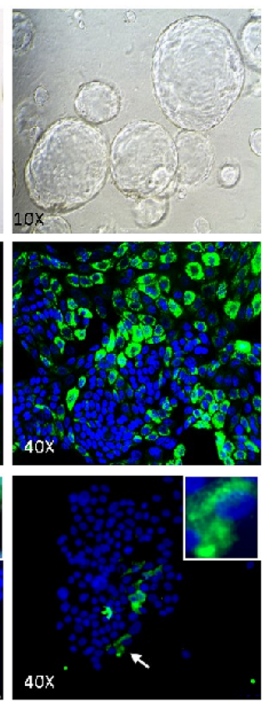

b

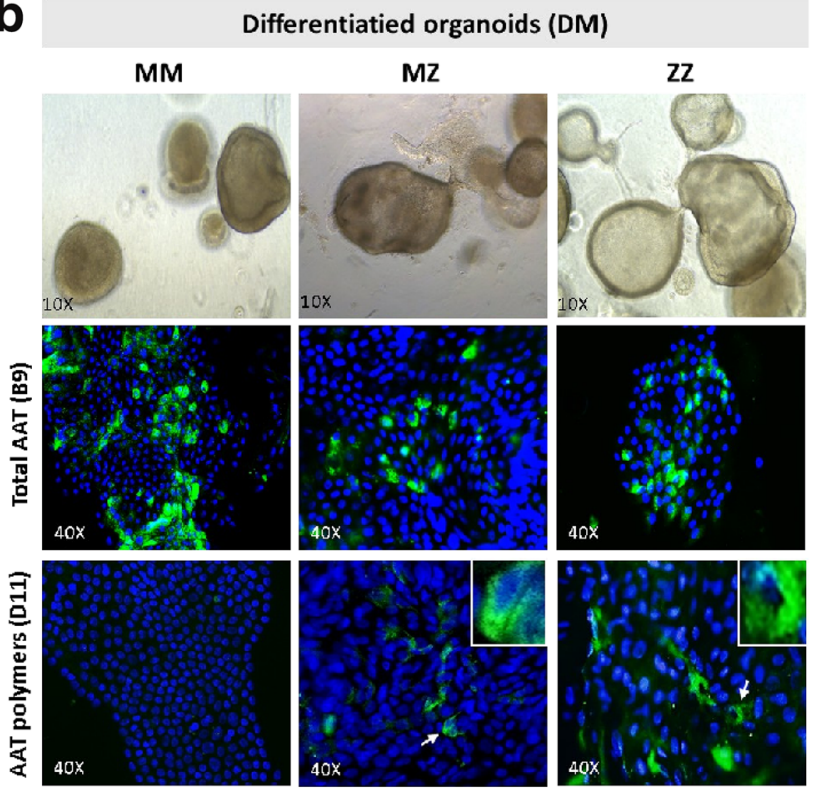

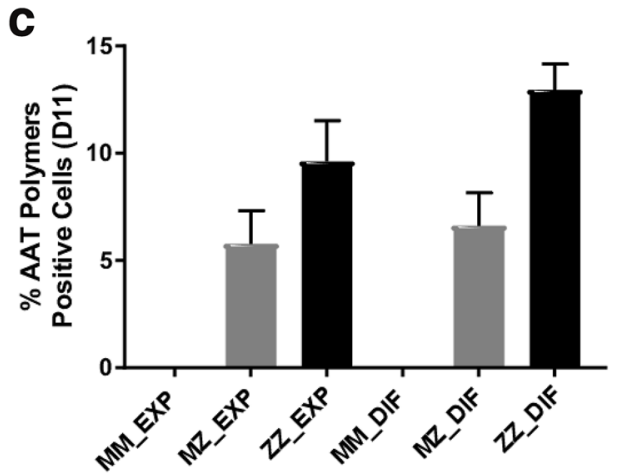

Fig. 5 Immunofluorescence detection of AAT and AAT polymers. a Representative pictures of liver organoids in the expansion medium $(\mathrm{EM})$ of $\mathrm{MM}, \mathrm{MZ}$ and $\mathrm{ZZ}$ patients (images magnification $\times 10$ ). $\mathbf{b}$ Differentiated liver organoids from MM, MZ and ZZ patients. Specific detection of total AAT protein with anti-AAT-B9 or with antiAAT-D11 against AAT polymers, are shown in green fluorescence.

good model to study the expression of liver AAT in relation to different AAT genotypes.

It is also important to point out that the expression of the STIC4 alternative short transcript of SERPINA1 gene was up-regulated by OSM. Interestingly, some differences were observed between $\mathrm{Z}$ and $\mathrm{M}$ organoids. Namely, higher induction of the STIC4 was found in MM and MZ than in $\mathrm{ZZ}$ organoids after hepatic cell differentiation. The expression of STIC4 was previously found in the liver [5]; however, the transcriptional regulation and functional role of this transcript are not clear. The induction of STIC4 transcript in response to inflammatory signal further confirms its functional role and encourages further investigations.
Zoomed images of individual positive cells are showed in the right corner. c Quantification of AAT polymers (D11) positive cells in the different organoids MM, MZ and ZZ. Microscope Zeiss Ax10, camera Axio Cam Mrm Carl Zeiss and software AxioVision Rel.4.7 (images magnification $\times 40$ )

Hypothetically, this transcript can generate C-terminal peptide of AAT, which has been found to regulate bile acid synthesis in primary rat hepatocytes in vivo [26].

Based on clinical and experimental data, we have learned that hepatocytes from Z-AAT deficiency carriers can be damaged by gain-of-toxic mechanisms activated because of intracellular accumulation of Z-AAT protein [27]. As expected, the cultures of hepatic organoids derived from patients carrying Z-AAT mutation revealed intrahepatic aggregates of misfolded Z-AAT protein. The intracellular AAT accumulation (positive PAS-D staining) and polymerization (immunostainings with specific anti-Z-AAT polymer antibody) were abundant in organoids derived from $\mathrm{ZZ}$ and also detected in $\mathrm{MZ}$ 


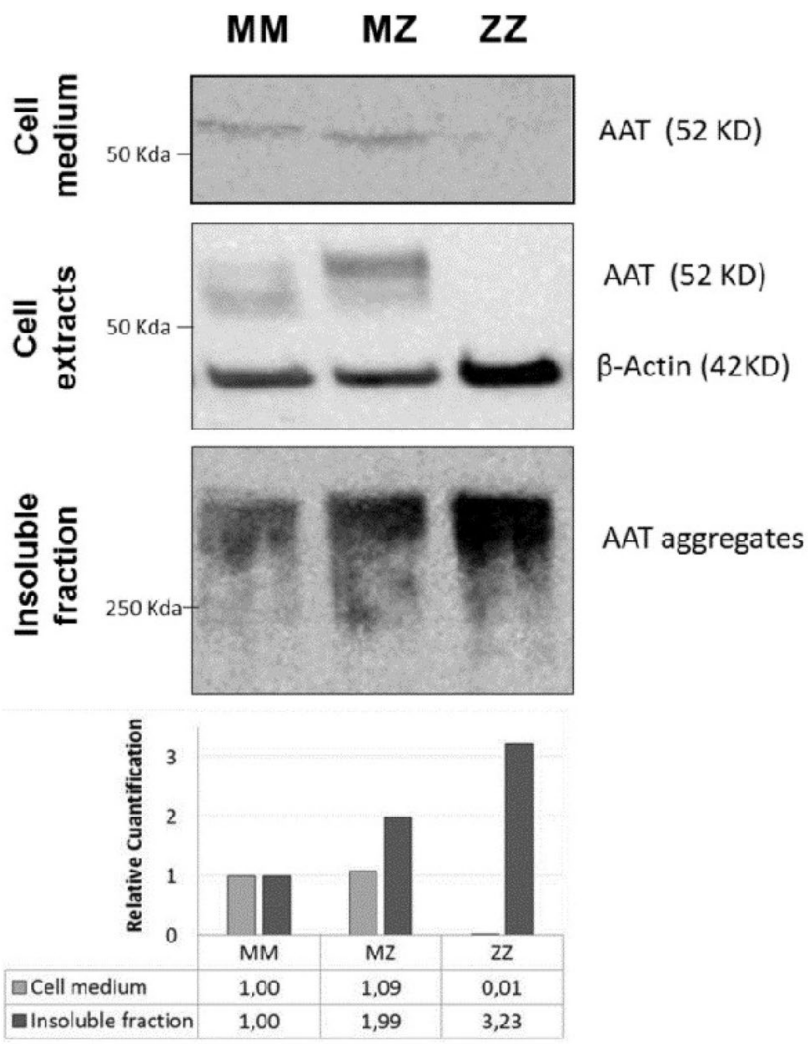

Fig. 6 Representative western blot detection of AAT protein expression and secretion in $\mathrm{MM}, \mathrm{MZ}$ and $\mathrm{ZZ}$ differentiated organoids. Blots of extracellular, cytoplasmic and insoluble fractions were incubated with anti-AAT-B9. The anti- $\beta$ actin antibody was used as a control marker in the cytoplasm extract. Panels of figures were composed to show MM, MZ and ZZ AAT in the same order. Relative quantification with respect to MM amount of AAT in the extracellular medium and in the insoluble fraction of organoids is shown at the bottom

subjects. Differently from MM and MZ organoid cultures, ZZ organoids showed almost no detectable secretion of AAT protein. These findings indicate that liver organoid cultures reflect AAT behavior seen in individuals with normal M- and mutant Z-AAT, and thus represent a valuable tool to study the molecular mechanisms underlying intracellular accumulation and secretion of AAT protein. Since organoids are derived directly from the affected individuals, they may also help us to elucidate the complex heterogeneity of liver disease among AATD carriers. In addition to Z-AAT mutation, many other rare alleles associated with AAT deficiency have been described $[19,28,29]$ for which the potential damaging effects in hepatocytes are unknown. Organoids derived from patients carrying other than the $\mathrm{Z}$ variant would help to investigate the mechanisms of accumulation, secretion and degradation of these much less studied AAT variants.

Moreover, the development of organoid cultures has been successfully reported for other organs derived from the endoderm, such as the intestine, stomach, pancreas and lung, as well as for other internal organs derived from the mesoderm including the kidney, heart, cartilage, bone, reproductive organs and muscle, and also for tissues derived from ectoderm, both skin and its associated glands and neural ectoderm, giving rise to the brain, spinal cord and neural crest [8]. Organoids have proved very useful for a wide range of applications such as modeling human diseases, organogenesis and tissue development, drug screening, gene editing for monogenic diseases and regenerative medicine $[8,30]$.

Organoids represent an innovative approach to validate specific genetic alterations in the SERPINA1 gene that are associated with liver disease development, and furthermore to identify and elucidate other genes associated with liver disease progression and to investigate putative treatments.

Acknowledgements This work was funded by grants from the Institute of Health Carlos III (AESI PI17CIII/00042 and DTS17CIII/00007) and Alpha-1 Antitrypsin Laurell's Training award, ALTA 2017. Supporting institutions had no involvement in the study design; collection, analysis and interpretation of data; writing of the manuscript; and decision to submit the manuscript for publication.

Authors' contributions GGM: established organoids, performed experiments, analyzed data, drafted manuscript. NM: established organoids, performed experiments, analyzed data, drafted manuscript. SM: performed experiments, analyzed data, IJ: provided samples, reviewed patient's clinical data, interpreted results AM: provided samples, reviewed patient's clinical data, interpreted results. CJ: provided samples, reviewed patient's clinical data, interpreted results. SM: performed bioinformatic analysis, helped in drafting manuscript. IC: performed bioinformatic analysis, helped in drafting manuscript. CG: provided samples, reviewed patient's clinical data, interpreted results. MTM: reviewed patient's clinical data, interpreted results. MH: provided samples, analyzed data, drafted the manuscript and revised it critically. IPC: analyzed data, drafted the manuscript and revised it critically. MP: participated in the collection of samples, interpreted the results, final review. SJ: participated in the study design, interpreted the results, drafted the manuscript. BMD: designed and coordinated the study, drafted the manuscript. All authors read and approved the final manuscript.

\section{Compliance with ethical standards}

Conflict of interest Gema Gómez-Mariano, Nerea Matamala, Selene Martínez, Iago Justo, Alberto Marcacuzco, Carlos Jimenez, Sara Monzón, Isabel Cuesta, Cristina Garfia, María Teresa Martínez, Meritxell Huch, Ignacio Pérez de Castro, Manuel Posada, Sabina Janciauskiene and Beatriz Martínez-Delgado declare no conflicts of interest.

Open Access This article is licensed under a Creative Commons Attribution 4.0 International License, which permits use, sharing, adaptation, distribution and reproduction in any medium or format, as long as you give appropriate credit to the original author(s) and the source, provide a link to the Creative Commons licence, and indicate if changes were made. The images or other third party material in this article are included in the article's Creative Commons licence, unless indicated otherwise in a credit line to the material. If material is not included in the article's Creative Commons licence and your intended use is not permitted by statutory regulation or exceeds the permitted use, you will need to obtain permission directly from the copyright holder. To view a copy of this licence, visit http://creativecommons.org/licenses/by/4.0/. 


\section{References}

1. Carlson JA, Rogers BB, Sifers RN, Hawkins HK, Finegold MJ, Woo SL. Multiple tissues express alpha 1-antitrypsin in transgenic mice and man. J Clin Invest. 1988;82(1):26-36.

2. Janciauskiene SM, Bals R, Koczulla R, Vogelmeier C, Kohnlein T, Welte T. The discovery of alpha1-antitrypsin and its role in health and disease. Respir Med. 2011;105(8):1129-39.

3. American Thoracic Society/European Respiratory Society statement. standards for the diagnosis and management of individuals with alpha-1 antitrypsin deficiency. Am J Respir Crit Care Med. 2003;168(7):818-900.

4. Sveger T. The natural history of liver disease in alpha 1-antitrypsin deficient children. Acta Paediatr Scand. 1988;77(6):847-51.

5. Fairbanks KD, Tavill AS. Liver disease in alpha 1-antitrypsin deficiency: a review. Am J Gastroenterol. 2008;103(8):2136-41.

6. Haugabook SJ, Ferrer M, Ottinger EA. In vitro and in vivo translational models for rare liver diseases. Biochim Biophys Acta Mol Basis Dis. 2019;1865(5):1003-18.

7. Huch M, Gehart H, van Boxtel R, Hamer K, Blokzijl F, Verstegen $\mathrm{MM}$, et al. Long-term culture of genome-stable bipotent stem cells from adult human liver. Cell. 2015;160(1-2):299-312.

8. Lancaster MA, Huch M. Disease modelling in human organoids. Dis Model Mech. 2019;12(7):dmm039347.

9. Nantasanti S, de Bruin A, Rothuizen J, Penning LC, Schotanus BA. Concise review: organoids are a powerful tool for the study of liver disease and personalized treatment design in humans and animals. Stem Cells Transl Med. 2016;5(3):325-30.

10. Lara B, Martinez-Delgado B, Torres ML, Marin-Arguedas S, Bustamante A, Miravitlles M. Alpha-1-antitrypsin deficiency associated with the Mattawa variant. Arch Bronconeumol. 2013;49(12):548-50.

11. Zorzetto M, Russi E, Senn O, Imboden M, Ferrarotti I, Tinelli C, et al. SERPINA1 gene variants in individuals from the general population with reduced alpha1-antitrypsin concentrations. Clin Chem. 2008;54(8):1331-8.

12. Broutier L, Andersson-Rolf A, Hindley CJ, Boj SF, Clevers H, Koo BK, et al. Culture and establishment of self-renewing human and mouse adult liver and pancreas 3D organoids and their genetic manipulation. Nat Protoc. 2016;11(9):1724-43.

13. Matamala N, Martinez MT, Lara B, Perez L, Vazquez I, Jimenez A, et al. Alternative transcripts of the SERPINA1 gene in alpha-1 antitrypsin deficiency. J Transl Med. 2015;4(13):211.

14. Matamala N, Aggarwal N, Iadarola P, Fumagalli M, GomezMariano G, Lara B, et al. Identification of novel short C-terminal transcripts of human SERPINA1 gene. PLoS One. 2017;12(1):e0170533.

15. Su AI, Cooke MP, Ching KA, Hakak Y, Walker JR, Wiltshire $\mathrm{T}$, et al. Large-scale analysis of the human and mouse transcriptomes. Proc Natl Acad Sci USA. 2002;99(7):4465-70.

16. Hsiao LL, Dangond F, Yoshida T, Hong R, Jensen RV, Misra J, et al. A compendium of gene expression in normal human tissues. Physiol Genomics. 2001;7(2):97-104.

17. Norris CA, He M, Kang LI, Ding MQ, Radder JE, Haynes MM, et al. Synthesis of IL- 6 by hepatocytes is a normal response to common hepatic stimuli. PLoS One. 2014;9(4):e96053.
18. Janciauskiene S, Eriksson S, Callea F, Mallya M, Zhou A, Seyama $\mathrm{K}$, et al. Differential detection of PAS-positive inclusions formed by the Z, Siiyama, and Mmalton variants of alpha1-antitrypsin. Hepatology. 2004;40(5):1203-10.

19. Matamala N, Lara B, Gomez-Mariano G, Martinez S, Retana D, Fernandez T, et al. Characterization of novel missense variants of SERPINA1 Gene causing alpha-1 antitrypsin deficiency. Am J Respir Cell Mol Biol. 2018;58(6):706-16.

20. Nibourg GA, Chamuleau RA, van Gulik TM, Hoekstra R. Proliferative human cell sources applied as biocomponent in bioartificial livers: a review. Expert Opin Biol Ther. 2012;12(7):905-21.

21. Tafaleng EN, Chakraborty S, Han B, Hale P, Wu W, Soto-Gutierrez A, et al. Induced pluripotent stem cells model personalized variations in liver disease resulting from alpha1-antitrypsin deficiency. Hepatology. 2015;62(1):147-57.

22. Yui S, Nakamura T, Sato T, Nemoto Y, Mizutani T, Zheng X, et al. Functional engraftment of colon epithelium expanded in vitro from a single adult $\operatorname{Lgr} 5(+)$ stem cell. Nat Med. 2012;18(4):618-23.

23. Henriksen JH, Siemssen O, Krintel JJ, Malchow-Moller A, Bendtsen F, Ring-Larsen H. Dynamics of albumin in plasma and ascitic fluid in patients with cirrhosis. J Hepatol. 2001;34(1):53-60.

24. Hamesch K, Mandorfer M, Pereira VM, Moeller LS, Pons M, Dolman GE, et al. Liver fibrosis and metabolic alterations in adults with alpha1 antitrypsin deficiency caused by the $\mathrm{Pi}$ *ZZ mutation. Gastroenterology 2019

25. Morgan K, Marsters P, Morley S, van Gent D, Hejazi A, Backx $M$, et al. Oncostatin $\mathrm{M}$ induced alpha1-antitrypsin (AAT) gene expression in Hep G2 cells is mediated by a $3^{\prime}$ enhancer. Biochem J. 2002;365(Pt 2):555-60.

26. Janciauskiene $\mathrm{S}$, Lindgren $\mathrm{S}$. Effects of fibrillar C-terminal fragment of cleaved alpha1-antitrypsin on cholesterol homeostasis in HepG2 cells. Hepatology. 1999;29(2):434-42.

27. Teckman JH, Qu D, Perlmutter DH. Molecular pathogenesis of liver disease in alpha1-antitrypsin deficiency. Hepatology. 1996;24(6):1504-16.

28. Renoux C, Odou MF, Tosato G, Teoli J, Abbou N, Lombard C, et al. Description of 22 new alpha-1 antitrypsin genetic variants. Orphanet J Rare Dis. 2018;13(1):161.

29. Giacopuzzi E, Laffranchi M, Berardelli R, Ravasio V, Ferrarotti I, Gooptu B, et al. Real-world clinical applicability of pathogenicity predictors assessed on SERPINA1 mutations in alpha-1-antitrypsin deficiency. Hum Mutat. 2018;39(9):1203-13.

30. Schwank G, Koo BK, Sasselli V, Dekkers JF, Heo I, Demircan $\mathrm{T}$, et al. Functional repair of CFTR by CRISPR/Cas9 in intestinal stem cell organoids of cystic fibrosis patients. Cell Stem Cell. 2013;13(6):653-8.

Publisher's Note Springer Nature remains neutral with regard to jurisdictional claims in published maps and institutional affiliations. 


\section{Affiliations}

\section{Gema Gómez-Mariano ${ }^{1}$ - Nerea Matamala ${ }^{1}$. Selene Martínez ${ }^{1} \cdot$ lago Justo ${ }^{2}$ - Alberto Marcacuzco ${ }^{2}$ - Carlos Jimenez ${ }^{2}$. Sara Monzón ${ }^{3} \cdot$ Isabel Cuesta ${ }^{3} \cdot$ Cristina Garfia ${ }^{4}$ María Teresa Martínez ${ }^{5} \cdot$ Meritxell Huch $^{6} \cdot$ Ignacio Pérez de Castro $^{7}$. Manuel Posada ${ }^{8} \cdot$ Sabina Janciauskiene ${ }^{9} \cdot$ Beatriz Martínez-Delgado ${ }^{1,8}$}

Gema Gómez-Mariano ggomezm@isciii.es

Nerea Matamala neremat@hotmail.com

Selene Martínez selenemr29@gmail.com

Iago Justo

iagojusto@hotmail.com

Alberto Marcacuzco

alejandro_mar@icloud.com

Carlos Jimenez

carlos.jimenez@inforboe.es

Sara Monzón

smonzon@isciii.es

Isabel Cuesta

isabel.cuesta@isciii.es

Cristina Garfia

cgarfia@hotmail.com

María Teresa Martínez

mmartinezm.hdoc@salud.madrid.org

Meritxell Huch

mh771@cam.ac.uk

Ignacio Pérez de Castro

iperez@isciii.es

Manuel Posada

mposada@isciii.es
Sabina Janciauskiene

sabinajanciauskiene@gmail.com

1 Molecular Genetics Unit, Institute of Rare Diseases Research, Institute of Health Carlos III (ISCIII), Ctra. Majadahonda-Pozuelo Km2,200, 28220 Madrid, Spain

2 General and Digestive Surgery Department, Hospital Doce de Octubre, Madrid, Spain

3 Bioinformatics Unit, Institute of Health Carlos III (ISCIII), Madrid, Spain

4 Digestive Department, Hospital Doce de Octubre, Madrid, Spain

5 Neumology Department, Hospital Doce de Octubre, Madrid, Spain

6 Wellcome Trust-Medical Research Council Stem Cell Institute, University of Cambridge, Cambridge, UK

7 Gene Therapy Unit, Institute of Rare Diseases Research, Institute of Health Carlos III (ISCIII), Madrid, Spain

8 Institute of Rare Diseases Research, Institute of Health Carlos III (ISCIII), Centre for Biomedical Network Research on Rare Diseases, CIBERER, Madrid, Spain

9 Department of Respiratory Medicine, German Centre for Lung Research (DZL), Hannover Medical School, Hannover, Germany 